Bull. Austral. Math. Soc.

VOL. 63 (2001) [101-104]

\title{
A METRISATION THEOREM FOR PSEUDOCOMPACT SPACES
}

\author{
Chris Good and A.M. Mohamad
}

In this paper we prove that a completely regular pseudocompact space with a quasiregular-G $G_{\delta}$-diagonal is metrisable.

\section{INTRODUCTION}

Recently, we have considered the question of what topological properties imply metrisability in the presence of a weak diagonal property. For example, it is well-known that the existence of a quasi- $G_{\delta}$-diagonal is sufficient for metrisability in countably compact spaces [7]. In [3] we proved that a manifold with a quasi-regular- $G_{\delta}-$ diagonal is metrisable. In this present paper, we give a diagonal condition on pseudocompact spaces to get metrisability.

A countable family $\left\{\mathcal{G}_{n}\right\}_{n \in \mathbb{N}}$ of collections of open subsets of a space $X$ is called a quasi-G⿰-diagonal (quasi-G $G_{\delta}^{*}$-diagonal), if for each $x \in X$ we have $\bigcap_{n \in c(x)} s t\left(x, \mathcal{G}_{n}\right)=\{x\}$ $\left(\bigcap_{n \in c(x)} \overline{\operatorname{st}\left(x, \mathcal{G}_{n}\right)}=\{x\}\right)$ where $c(x)=\left\{n: x \in G\right.$ for some $\left.G \in \mathcal{G}_{n}\right\}$ and $s t\left(x, \mathcal{G}_{n}\right)$ is the union of all sets in $\mathcal{G}_{n}$ which contain $x$.

A space $X$ has a quasi-regular- $G_{\delta}$-diagonal [3] if and only if there is a countable sequence $\left\langle U_{n}: n \in \mathbb{N}\right\rangle$ of open subsets in $X^{2}$, such that for all $(x, y) \notin \Delta$, there is $n \in \mathbb{N}$ such that $(x, x) \in U_{n}$ but $(x, y) \notin \overline{U_{n}}$.

A space $X$ is called quasi-developable if there is a countable family $\left\{\mathcal{G}_{n}: n \in \mathbb{N}\right\}$ of collections of open subsets of $X$ such that for all $x \in X$ the nonempty sets of the form $s t\left(x, \mathcal{G}_{n}\right)$ form a local base at $x$.

In this paper all spaces will be completely regular, unless we state otherwise.

\section{THE MAIN RESULTS}

Pseudocompact spaces were first defined and investigated by Hewitt in [4].

DEFINITION 2.1. A space $X$ is pseudocompact if every real-valued continuous function on $X$ is bounded.

The following characterisation of pseudocompactness may be found in [2].

Received 6th August, 2000

Copyright Clearance Centre, Inc. Serial-fee code: 0004-9727/01 \$A2.00+0.00. 
LEMMA 2.2. A space $X$ is pseudocompact if and only if for every decreasing sequence $\left\langle U_{n}: n \in \mathbb{N}\right\rangle$ of nonvoid open subsets of $X, \bigcap_{n \in \mathbb{N}} \overline{U_{n}} \neq \emptyset$.

McArthur in [6] proved the following lemma.

Lemma 2.3. Let $X$ be a pseudocompact space. Suppose $\left\langle U_{n}: n \in \mathbb{N}\right\rangle$ is a decreasing sequence of open sets such that $\bigcap_{n \in \mathbb{N}} U_{n}=\bigcap_{n \in \mathbb{N}} \overline{U_{n}}=\{x\}$ for a point $x \in X$. Then the sets $U_{n}$ form a local neighbourhood base at $x$.

The proof of our main result relies on a metrisation theorem.

THEOREM 2.4. [3] Let $X$ be a space with a sequence $\left\langle\mathcal{G}_{n}: n \in \mathbb{N}\right\rangle$ of open families such that, for each $x \in X,\left\{s t^{2}\left(x, \mathcal{G}_{n}\right)\right\}_{n \in \mathbb{N}}-\{\emptyset\}$ (that is, the union of all sets $\operatorname{st}\left(y, \mathcal{G}_{n}\right)$ with $\left.y \in \operatorname{st}\left(x, \mathcal{G}_{n}\right)\right)$ is a local base at $x$. Then $X$ is metrisable.

LEMMA 2.5. Let $X$ be a pseudocompact space with a quasi-G*-diagonal. Then $X$ is quasi-developable.

Proof: Let $\left\langle\mathcal{V}_{n}: n \in \mathbb{N}\right\rangle$ be a quasi-G*-diagonal sequence for $X$. Without loss of generality we may assume that $\mathcal{V}_{1}=\{X\}$. Set $\mathcal{C}_{V}(x)=\left\{n:\right.$ st $\left.\left(x, \mathcal{V}_{n}\right) \neq \emptyset\right\}$. Then $\bigcap \overline{\operatorname{st}\left(x, \mathcal{V}_{n}\right)}=\{x\}$. Let $\mathcal{F}$ denote the set of non-empty finite subsets of $\mathbb{N}$. For each $n \in \mathcal{C}_{\mathcal{V}}(x)$ $F \in \mathcal{F}$ set

$$
\mathcal{G}_{F}=\left\{\bigcap_{i \in F} V_{i}: V_{i} \in \mathcal{V}_{i}\right\}
$$

We show that $\left\{\mathcal{G}_{F}: F \in \mathcal{F}\right\}$ is a quasi-development of $X$. For each $n \in \mathbb{N}, x \in X$ put $F_{n}(x)=c_{\mathcal{V}}(x) \cap\{1,2, \ldots, n\}$. Then $F_{n}(x) \neq \emptyset$. Note that $\operatorname{st}\left(x, \mathcal{G}_{F_{n}(x)}\right) \subseteq \operatorname{st}\left(x, \mathcal{V}_{m}\right)$ for each $n \in \mathbb{N}$, each $x \in X$ and each $m \in F_{n}(x)$. Note also that

$$
\bigcap_{n \in \mathbb{N}} \overline{s t\left(x, \mathcal{G}_{F_{n}(x)}\right)}=\bigcap_{n \in \mathbb{N}} s t\left(x, \mathcal{G}_{F_{n}(x)}\right)=\{x\} .
$$

By Lemma 2.3, $\left\{s t\left(x, \mathcal{G}_{F_{n}(x)}\right): n \in \mathbb{N}\right\}$ forms a local neighbourhood base at $x$. Hence, $\left\{s t\left(x, \mathcal{G}_{F}\right): F \in \mathcal{F}\right\}-\emptyset$ forms a local neighborhood base at $x$.

THEOREM 2.6. Let $X$ be a pseudocompact space with a quasi-regular- $G_{\delta^{-}}$ diagonal. Then $X$ is metrisable.

ProOF: By Theorem 2.4, we only need to show that $X$ has a quasi-development $\left\langle\mathcal{G}_{n}: n \in \mathbb{N}\right\rangle$ such that, for each $x \in X,\left\{s t^{2}\left(x, \mathcal{G}_{n}\right)\right\}_{n \in \mathbb{N}}-\{\emptyset\}$ is a local base at $x$.

Let $\left\langle U_{n}: n \in \mathbb{N}\right\rangle$ be as in the definition of quasi-regular- $G_{\delta}$-diagonal. So, the sets $U_{n}$ are open in $X^{2}$ and for all $(x, y) \notin \Delta$, there is $n \in \mathbb{N}$ such that $(x, x) \in U_{n}$ but $(x, y) \notin \bar{U}_{n}$. Put $\mathcal{H}_{n}=\left\{H: H\right.$ is open , $\left.H \times H \subseteq U_{n}\right\}$. As in the proof of Lemma 2.5, let $\mathcal{F}$ denote the set of non-empty finite subsets of $\mathbb{N}$, and for $F \in \mathcal{F}$ put

$$
\mathcal{G}_{F}^{\prime}=\left\{\bigcap_{i \in F} H_{i}: H_{i} \in \mathcal{H}_{i}\right\}
$$


We show that for each $x \in X,\left\{s t^{2}\left(x, \mathcal{G}_{F}^{\prime}\right)\right\}_{F \in \mathcal{F}}-\{\emptyset\}$ is a local base at $x$. Take any $x \in X$. For each $n \in \mathbb{N}$ put $F_{n}(x)=\left\{i: s t\left(x, \mathcal{H}_{i}\right) \neq \emptyset\right\} \cap\{1,2, \ldots, n\}$. Without loss, $\mathcal{H}_{i}=\{X\}$, so $F_{n}(x) \neq \emptyset$. We prove that $\bigcap_{n \in \mathbb{N}} \overline{s t^{2}\left(x, \mathcal{G}_{F_{n}(x)}^{\prime}\right)}=\{x\}$.

Suppose, for a contradiction, for all $n \in \mathbb{N}, y \in \overline{s t^{2}\left(x, \mathcal{G}_{F_{n}(x)}^{\prime}\right)}$ and $x \neq y$. So by the definition of quasi-regular- $G_{\delta}$-diagonal, there is $k$ such that $(x, x) \in U_{k}$ but $(x, y) \notin \bar{U}_{k}$.

By the same argument as in Lemma 2.5 , we know that $\left\{\mathcal{G}_{F}^{\prime}: F \in \mathcal{F}\right\}$ is a quasidevelopment of $X$. Therefore there exist $I$ and $J \in \mathcal{F}$ such that

$$
(x, y) \in \operatorname{st}\left(x, \mathcal{G}_{\mathrm{f}}^{\prime}\right) \times \operatorname{st}\left(y, \mathcal{G}_{\mathrm{J}}^{\prime}\right) \subseteq X^{2}-\bar{U}_{n} .
$$

Choose $m \geqslant \max \{I, k\}$, so that $I \subseteq F_{m}(x)$. It follows that $y \in \overline{s t^{2}\left(x, \mathcal{G}_{F_{m}(x)}^{\prime}\right)}$, so $s t^{2}\left(x, \mathcal{G}_{F_{m}(x)}^{\prime}\right) \cap s t\left(y, \mathcal{G}_{J}^{\prime}\right) \neq \emptyset$. Then there exists $G_{1}, G_{2} \in \mathcal{G}_{F_{m}(x)}^{\prime}$ and $G_{3} \in \mathcal{G}_{J}^{\prime}$ such that $y \in G_{3}, x \in G_{1}, G_{1} \cap G_{2} \neq \emptyset$ and $G_{2} \cap G_{3} \neq \emptyset$. Let $z_{1} \in G_{1} \cap G_{2}$ and $z_{2} \in G_{2} \cap G_{3}$. Then $\left(z_{1}, z_{2}\right) \in\left(G_{1} \times G_{3}\right) \cap\left(G_{2} \times G_{2}\right)$. Now, $G_{1} \in \mathcal{G}_{F_{m}(x)}^{\prime}, G_{3} \in \mathcal{G}_{J}^{\prime}$, so $G_{1} \times G_{3} \subseteq \operatorname{st}\left(x, \mathcal{G}_{F_{m}(x)}^{\prime}\right) \times s t\left(y, \mathcal{G}_{J}^{\prime}\right)$. Also, $G_{2} \in \mathcal{G}_{F_{m}(x)}^{\prime}$ and $k \in F_{m}(x)$, so $G_{2} \subseteq H$ for some $H \in \mathcal{H}_{k}$. Therefore $G_{2} \times G_{2} \subseteq H \times H \subseteq U_{k}$, so $\left(z_{1}, z_{2}\right) \in U_{k}$.

In other words, $\left(z_{1}, z_{2}\right) \in\left(G_{2} \times G_{3}\right) \cap U_{k} \subseteq\left(s t\left(x, \mathcal{G}_{F_{m(x)}}^{\prime}\right) \times s t\left(y, \mathcal{G}_{J}^{\prime}\right)\right) \cap U_{k}$, and this is a contradiction. Therefore, $\bigcap_{n \in \mathcal{C}_{\mathcal{G}^{\prime}}(x)} \overline{s t^{2}\left(x, \mathcal{G}_{F_{n}(x)}^{\prime}\right)}=\{x\}$. We conclude by Lemma 2.3 that for each $x \in X,\left\{s t^{2}\left(x, \mathcal{G}_{F}^{\prime}\right)\right\}_{F \in \mathcal{F}}-\{\emptyset\}$ is a local base at $x$. Hence, $X$ is metrisable.

EXAMple 2.7. The space $E \cap[0,1]$ of [2, Problem 3J] is submetrisable (that is, it is a space with a coarser metric topology) pseudocompact and Hausdorf. Since the space is not completely regular, it is not metrisable.

EXAMPLE 2.8. The Mrowka space $\Psi$ (see $[2,1,5]$ ) is completely regular, pseudocompact and developable but does not have a quasi-regular- $G_{\delta}$-diagonal, and hence is not metrisable.

\section{REFERENCES}

[1] D. Burke, 'Covering properties', in Handbook of Set-Theoretic Topology (North-Holland, Amsterdam, New York, 1984), pp. 347-422.

[2] L. Gillman and M. Jerison, Rings of continuous functions (Van Nostrand, Princeton, N.J., 1960).

[3] P.M. Gartside and A.M. Mohamad, 'Cleavability of manifolds', Topology Proc. 23 (1998), 151-166.

[4] E. Hewitt, 'Rings of real-valued continuous functions. I', Trans. Amer. Math. Soc. 64 (1948), 45-99.

[5] K. Lee, 'Spaces in which compacta are uniformly regular $G_{\delta}$ ', Pacific J. Math. 81 (1979), 435-445.

[6] W. McArthur, ' $G_{\delta}$-diagonals and metrization theorems', Pacific J. Math. 44 (1973), 613-617. 
[7] H. Shiraki, 'A note on spaces with a uniform base', Proc. Japan. Acad. Ser. A Math. Sci 47 (1971), 1036-1041.

School of Mathematics and Statistics University of Birmingham Birmingham, B15 2TT United Kingdom e-mail: c.good@bham.ac.uk.
Department of Mathematics and Statistics

College of Science

Sultan Qaboos University

Muscat, Oman

e-mail: mohamad@squ.edu.om 